\title{
Experiments Measuring Particle Deposition from Fully Developed Turbulent Flow in Ventilation Ducts
}

\author{
MARK R. SiPPOLA and William W. NAZAROFF ${ }^{*}$ \\ Indoor Environment Department, Environmental Energy Technologies Division, Ernest Orlando \\ Lawrence Berkeley National Laboratory, Berkeley, CA 94720 USA \\ Department of Civil and Environmental Engineering, University of California, Berkeley, CA \\ 94720-1710 USA
}

\section{Abstract}

Particle deposition in ventilation ducts influences particle exposures of building occupants and may lead to a variety of indoor air quality concerns. Experiments have been performed in a laboratory to study the effects of particle size and air speed on deposition rates of particles from turbulent air flows in galvanized steel and internally insulated ducts with hydraulic diameters of $15.2 \mathrm{~cm}$. The duct systems were constructed of materials typically found in commercial heating, ventilating and air conditioning (HVAC) systems. In the steel duct system, experiments with nominal particle sizes of $1,3,5,9$ and $16 \mu \mathrm{m}$ were conducted at each of three nominal air speeds: 2.2, 5.3 and $9.0 \mathrm{~m} / \mathrm{s}$. In the insulated duct system, deposition rates of particles with nominal sizes of $1,3,5,8$ and $13 \mu \mathrm{m}$ were measured at nominal air speeds of 2.2, 5.3 and $8.8 \mathrm{~m} / \mathrm{s}$. Fluorescent techniques were used to directly measure the deposition velocities of monodisperse fluorescent particles to duct surfaces (floor, wall and ceiling) at two straight duct sections where the turbulent flow profile was fully developed.

In steel ducts, deposition rates were higher to the duct floor than to the wall, which were, in turn, greater than to the ceiling. In insulated ducts, deposition was nearly the same to the duct 
floor, wall and ceiling for a given particle size and air speed. Deposition to duct walls and ceilings was greatly enhanced in insulated ducts compared to steel ducts. Deposition velocities to each of the three duct surface orientations in both systems were found to increase with increasing particle size or air velocity over the ranges studied. Deposition rates measured in the current experiments were in general agreement with the limited observations of similar systems by previous researchers.

\section{Introduction}

Inhalation of airborne particles may adversely affect human health. Ventilation ducts are usually the dominant entry path of outdoor air into large buildings and indoor air is often circulated through ventilation ducts to be mixed with outdoor air, thermally conditioned, and returned to the indoor space. Most indoor air in large buildings passes through a ventilation duct at least once, and some air passes through several times. As air flows through ducts, deposition to duct surfaces may alter airborne particle size distributions and therefore affect exposures of building occupants.

If bioaerosols deposit, subsequent growth or biochemical transformations can lead to the release into ventilation air of a variety of indoor air pollutants such as mold spores and microbial volatile organic compounds. A better understanding of particle behavior in ventilation ducts is needed to evaluate pollutant exposures within buildings and contamination of HVAC systems.

Because of its importance to numerous systems, particle deposition from turbulent flow has been widely studied experimentally and theoretically. Airborne particles deposit to surfaces from turbulent flows by a variety of mechanisms. Factors known to influence deposition rates include particle size, degree of air turbulence, surface roughness and the orientation of the deposition surface with respect to gravity. While there is a large body of experimental literature 
on the general topic of particle deposition from turbulent flow, the specific case of deposition in ventilation ducts has not been substantially addressed. Horizontal floors, vertical walls and horizontal ceilings are common in nearly all runs with rectangular ducts. Most experiments in ducts with diameters and air speeds similar to those found in HVAC systems have only measured deposition to floors (Chamberlain et al., 1984; Kvasnak et al., 1993; Lai (1997)). Sehmel (1973) measured deposition rates to a duct ceiling that were one to two orders of magnitude less than to a floor. While most particle deposition in ducts is likely to occur at floors, information on deposition to duct walls and ceilings is necessary for a more complete understanding of deposition from turbulent flows.

The microscale roughness of the deposition surface may influence particle deposition rates. Theoretical analyses suggest that roughness elements of even a few microns in size could dramatically increase deposition (Browne, 1974; Fan \& Ahmadi, 1993). The experiments of ElShobokshy (1983) showed orders of magnitude increases in deposition when the deposition surface roughness was increased. The experimental work of Wells \& Chamberlain (1967) and Sehmel (1970) showed high deposition rates to fibrous surfaces compared to metallic surfaces.

Ducts in commercial buildings are usually made of galvanized steel. Commonly, some ducts have internal fiberglass insulation to reduce noise transmission and to provide a thermal barrier. The insulation generally has a composite layer exposed to the flow to prevent insulation fibers from being entrained into the airflow. Ducts may also be made of fiberglass duct board, corrugated aluminum and flexible spiral-wound Mylar. Particle deposition rates are likely to be strongly influenced by the type of internal duct surface.

In this paper, experiments to investigate the influence of particle size, air speed and deposition surface characteristics on particle deposition rates in HVAC ducts are described. The 
experiments were conducted in two experimental systems with the same layout: a galvanized steel duct system and an internally insulated duct system. Duct materials and air speeds investigated are common to commercial building HVAC systems and particle deposition was directly measured on the floors, walls and ceilings of the experimental duct surfaces. We report results for deposition rates in straight duct sections where the turbulent flow profile was fully developed. Models developed from these experimental results have been applied to understand the implications regarding particle fates in buildings and particle exposure evaluations (Sippola \& Nazaroff, 2003).

\section{Methods}

\subsection{Definitions}

The deposition velocity, $V_{d}$, of a particle to a duct surface is defined as

$$
V_{d}=\frac{J}{C_{a v e}}
$$

where $J$ is the time-averaged particle flux to the surface and $C_{\text {ave }}$ is the time-averaged airborne particle concentration in the bulk air passing through the duct.

The dimensionless deposition velocity, $V_{d}^{+}$, is defined as follows:

$$
V_{d}^{+}=\frac{V_{d}}{u^{*}}
$$

where $u^{*}$, the friction velocity of a turbulent duct flow, is calculated by this expression:

$$
u^{*}=U_{\text {ave }} \sqrt{f / 2}
$$

Here, $U_{\text {ave }}$ is the average air speed in the duct and $f$ is the Fanning friction factor. For fully developed turbulent flow, $f$ is given by 


$$
f=\frac{\Delta P}{\Delta L} \frac{D_{h}}{2 \rho_{a} U_{a v e}^{2}}
$$

where $\Delta P / \Delta L$ is the pressure drop per unit duct length and $\rho_{a}$ is the air density. The hydraulic diameter, $D_{h}$, is defined in the following manner:

$$
D_{h}=\frac{4 A}{p}
$$

Here, $A$ is the cross sectional area of the duct and $p$ is the perimeter of a section through the duct, normal to the flow direction. In the experiments reported here, the friction velocity was determined for a known air speed and duct dimensions by means of measuring the pressure drop and applying equations (3) and (4).

Neglecting particle slip (appropriate, since we are studying supermicron particle deposition at ambient pressure and temperature), a dimensionless particle relaxation time, $\tau^{+}$, may be calculated for spherical particles in the Stokes flow regime as follows:

$$
\tau^{+}=\frac{\rho_{p} d_{p}^{2} u^{* 2}}{18 \mu v}
$$

where $\rho_{p}$ is the particle density, $d_{p}$ is the particle diameter, $\mu$ is the dynamic viscosity of air and $v$ is the kinematic viscosity of air.

\subsection{Overview of Experimental Approach}

Particle deposition velocities were measured in both a steel-duct and an insulated duct system. The steel system is described first, and then differences between the two systems are discussed. Further details on the experimental methods are reported elsewhere (Sippola, 2002a). In the steel system, experiments were performed at three nominal air speeds of 2.2, 5.3 and 9.0 $\mathrm{m} / \mathrm{s}$ for each of five nominal particle diameters: $1,3,5,9$ and $16 \mu \mathrm{m}$. Including one replicate 
experiment, a total of sixteen experiments were conducted in the steel system (runs 1-16). In the insulated system, experiments were performed at three nominal air speeds $-2.2,5.3$ and $8.8 \mathrm{~m} / \mathrm{s}$ — for each of five nominal particle diameters: 1, 3, 5, 8 and $13 \mu \mathrm{m}$. Fifteen distinct experiments were conducted in the insulated system (runs 17-31).

\subsection{Steel duct apparatus}

Figure 1 presents a schematic of the experimental apparatus. Recirculating airflow was induced by means of a fan (Dayton Mfg., Model 4C108) with an adjustable speed, 1-hp 3-phase motor (Dayton Mfg.). Monodisperse experimental particles tagged with a fluorescent tracer (sodium fluorescein) were injected into the duct system at the mixing box. Particles were produced by means of a vibrating orifice aerosol generator (TSI Inc., Model 3450) and a Boltzmann charge distribution was imparted on the aerosol by passing it through a neutralizer (TSI Inc., Model 3054) with a Kr-85 radioactive source. Particles were generated from a liquid solution of isopropyl alcohol, oleic acid, and solid fluorescein dissolved in $0.1 \mathrm{M}$ aqueous ammonium hydroxide. Particle larger than $1 \mu \mathrm{m}$ comprised a suspension of ammonium fluorescein in oleic acid; $1 \mu \mathrm{m}$ particles were solid ammonium fluorescein.

Particle deposition fluxes were measured at test ducts 1 and 2. The lower and upper ducts consisted of several $1.5 \mathrm{~m}(5.0 \mathrm{ft})$ long galvanized steel sections with $15.2 \times 15.2 \mathrm{~cm}(6.0 \times$ 6.0 in) cross-sections (Allied Mechanical HVAC). The inlet to test duct 1 was $12.2 \mathrm{~m}$ (40.0 ft) downstream of the mixing box, giving a length of 80 hydraulic diameters for the turbulent flow profile to develop. Test duct 2 was 90 hydraulic diameters downstream of any flow disturbance.

Time-averaged airborne particle concentrations were measured immediately upstream and downstream of the test ducts. Isokinetic nozzles (Apex Instruments) continuously delivered aerosol from the duct centerline to $47 \mathrm{~mm}$ nitrocellulose filters (Osmonics) for the duration of an 
experiment. An automatic data acquisition board (Adac, Series 5000) controlled the pressure sensors (Setra, Model 264) at locations A and C. An Aerodynamic Particle Sizer (APS) (TSI Inc, Model 3320) continuously sampled the experimental aerosol at the centerline of the lower duct through a specially designed, shrouded sampling nozzle. Aerodynamic particle size data collected by the APS were used to calculate the mean physical diameter of experimental particles during each experiment.

\subsection{Experimental protocol with the steel system}

A well-defined protocol was followed for each experiment. The steps involved in conducting a single experiment were as follows:

1. On the day preceding the start of an experiment, test ducts, sampling nozzles, and filter holder assemblies were cleaned and dried.

2. On the first day of the experiment, sampling nozzles and test ducts were installed.

3. The fan was turned on and set to the proper speed. Measurements of the centerline air velocity pressures were made at the sampling nozzle locations and the sampling pump flow rates were adjusted to achieve isokinetic sampling.

4. Bulk air velocities in the upper and lower duct were measured.

5. Filters were inserted into sampling nozzles, which were attached to sampling pumps.

6. Fluorescent experimental particles were generated and injected into the system.

7. The APS was attached to the shrouded aerosol probe and programmed to collect aerodynamic particle size data for the duration of the experiment.

8. After sufficient time had elapsed, the particle generator was turned off.

9. Centerline air velocity pressures were measured at the sampling nozzle locations and sampling pump flow rates were measured. 
10. The fan, APS and sampling pumps were turned off and all test ducts, sampling nozzles, filter holders and filters were removed for wet chemistry analysis.

11. Wet chemistry was used to measure the particle mass deposited on filters, inside filter holders and inside sampling nozzles to determine airborne particle concentrations.

12. Wet chemistry was used to measure the particle mass deposited on duct surfaces to help determine particle deposition fluxes.

All air velocity determinations were made by means of a pitot tube (Dwyer Instruments Inc., Model H-11) attached to a digital handheld manometer (Energy Conservatory, Model DG3) to measure the air velocity pressure. Two types of air velocities were measured during experiments: centerline air velocities and bulk air velocities. Centerline air velocities were measured in step 3 at each sampling nozzle to determine the proper sampling pump flow rates to achieve isokinetic sampling. Continuously adjustable sampling pumps and a mass flow controller (Sierra, Model Sidetrack III) drew air through the sampling nozzles at the isokinetic flow rate. Pump flow rates were measured using an electronic bubble flow meter (Gilian Instruments, Gilibrator). Bulk air velocities were measured immediately upstream of test ducts 1 and 2 in step 4 using the equal area method with a grid of 16 points. The value of $U_{\text {ave }}$ reported for an experiment is the average of these two realizations.

Before injecting the aerosol into the mixing box, particle size and monodispersity were monitored with an APS. Once particles were input to the system, experimental run times (steps 6-8) varied in the range $4 \mathrm{~h}-6 \mathrm{~d}$ depending on the particle size and air speed.

Airborne particle concentrations and particle surface fluxes were quantified by fluorescent techniques in a wet-chemistry lab. Filters, duct surface panels and interior portions of nozzles and filter holders were each rinsed with a known amount of rinsing solution in a glass 
tray or Pyrex beaker. The fluorescence of the resulting wash solution was measured by means of a fluorometer (Turner Designs, Model TD-700).

Not all particles entering the isokinetic sampling nozzle reach the filter; some deposit on the nozzle interior or in the filter holder. To accurately determine the airborne concentration, the particle mass at these additional locations was measured and used in the concentration determination. Filters were rinsed by soaking in a known amount of rinsing solution. Filter holders and nozzles were rinsed by repeatedly passing a small amount of rinsing solution from a measured quantity through the piece by means of small-volume disposable transfer pipettes. Airborne concentrations were determined through knowledge of the total particle mass entering the nozzle, the experimental time and the average sampling pump flow rate.

Panels to be analyzed for particle deposition were cut from the test ducts using electric sheet metal shears (Kett Tools, Model K200). For each test duct, twelve panels were removed and analyzed: four panels each from the duct floor, sidewall and ceiling. The four panels from each duct surface were centered at locations that were $0.30,0.61,0.91$ and $1.22 \mathrm{~m}(1.0,2.0,3.0$ and $4.0 \mathrm{ft}$ ) from the test duct inlet and were labeled as shown in Figure 2. Cut panels were rectangular and typically measured about $0.1 \times 0.2 \mathrm{~m}$. After flourescence analysis, panels were measured to determine their actual lengths and widths. The particle mass flux to a duct surface was calculated from the measured particle mass on the panel, panel surface area and experimental time.

Deposition velocities in test ducts 1 and 2 were calculated by equation (1). The concentration for deposition velocity calculations was taken as the average of the concentration measurements made immediately upstream and downstream of the test duct. Particle deposition fluxes to four panels at each of the floor, wall and ceiling surfaces of both test ducts 1 and 2 were 
measured in each experiment. The reported deposition velocity to a given surface is the average of the deposition velocities measured at the four panels of that surface.

Data collected by the APS were converted from aerodynamic sizes to physical sizes and weighted averaging was used to determine the mass-mean diameter of the particles.

Dimensionless particle relaxation times were calculated by equation (6).

\subsection{Blank experiments: Detection limits and particle resuspension}

Blank experiments were conducted following the above procedures except that fluorescent particles were not generated or injected into the experimental duct. These blank experiments were used to determine the detection limits of the methods. In these blank experiments, the fluorescein masses on the air filters, isokinetic sampling nozzles and filter holders were all typically $0.0 \mathrm{ng}$. The measured masses of fluorescein on the duct panels during blank experiments were typically in the range $0-2 \mathrm{ng}$. The minimum fluorescein mass that could reliably be measured above background on a duct panel was taken to be $4 \mathrm{ng}$. When experiments with particles were conducted, the measured fluorescein mass on duct panels typically ranged from hundreds to tens of thousands of nanograms. The minimum amount of fluorescent material that was collected on a surface during any of the particle experiments was about $10 \mathrm{ng}$ (on ceiling panels of ducts during experiments with $1 \mu \mathrm{m}$ particles).

Blank experiments were conducted at air speeds of 2.2, 5.3 and $9.0 \mathrm{~m} / \mathrm{s}$. While conducting blank experiments, it was discovered that certain fluorescent experimental particles generated in previous experiments could resuspend into the air stream and redeposit onto the cleaned test duct surfaces. Specifically, resuspension was observed at air speeds of 5.3 and 9.0 $\mathrm{m} / \mathrm{s}$ for particles that were $5 \mu \mathrm{m}$ in diameter and larger. Resuspension of 1 or $3 \mu \mathrm{m}$ particles was not detected, nor was resuspension of any particles at air speeds of $2.2 \mathrm{~m} / \mathrm{s}$. 
Because of the observed resuspension of particles, experiments were conducted in a systematic pattern to reduce the possibility that resuspension could influence the results. Thus, experiments using particles of the same nominal size were conducted in succession at the three air speeds, ordered low to high. After three experiments with the same particle size, the entire duct system was dismantled, cleaned and dried and reassembled. Blank experiments conducted after this cleaning showed no signs of resuspension of residual fluorescent particles. Data collected by the APS also showed no signs of resuspension. After cleaning, three more experiments were conducted for the next larger particle size, and then the entire system was cleaned again. This process was repeated for each particle size.

\subsection{Insulated duct apparatus}

The internally insulated duct system had the same configuration shown in Figure 1 for the steel system and all equipment except for the ductwork was the same. The ducts were steel with $20.3 \times 20.3 \mathrm{~cm}(8.0 \times 8.0$ in $)$ cross-sections and $2.5 \mathrm{~cm}(1.0$ in $)$ thick acoustic fiberglass insulation on all interior surfaces. Thus, the flow area in the insulated ducts was $15.2 \times 15.2 \mathrm{~cm}$ $(6.0 \times 6.0 \mathrm{in})$, the same as in the steel system. The insulation (Certainteed, ToughGard R) was fiberglass overlaid with a fibrous black composite surface on the airstream side.

\subsection{Experimental protocol with the insulated system}

Experiments in the insulated duct system followed the same steps as outlined in section 2.4 for the steel duct system with some minor modifications. In step 1, only sampling nozzles and filter holder assemblies were pre-cleaned; test ducts could not readily be cleaned owing to the presence of the insulation. Measurements showed that the background fluorescein mass on 
insulated duct panels was in the range $0-4 \mathrm{ng}$; this level was sufficiently low for use in experiments, as the fluorescein mass on insulation panels was typically thousands of nanograms.

When particle masses on the insulated duct surfaces were determined in step 12, the panels were the black composite layer of the insulation that was exposed to the airflow, not the steel part of the duct. These panels were obtained by cutting open the test duct, and then slicing out panels with a handheld razor blade from locations as depicted in Figure 2.

The entire insulated system could not be disassembled and cleaned between experiments because of the presence of interior insulation in the ducts. Blank experiments suggested that residual fluorescent particles deposited in the insulated ducts would not significantly influence results. The minimum mass that could be reliably detected on an insulation panel was $10 \mathrm{ng}$. The minimum mass detected on an insulation panel in an experiment was about $200 \mathrm{ng}$. As in the steel system, APS data collected during experimental runs showed no signs of resuspension of previously deposited experimental particles. The same order of experiments, where three experiments with particles of the same size were conducted before proceeding to experiments with the next largest particle size, was followed for both the steel and insulated systems.

\subsection{Model predictions}

Particle deposition models provide estimates of deposition rates given information about the particles, the airflow and the airflow conduit. Common input information for most models includes the particle size and density, the air velocity, and the duct dimensions. Some models predict the influence of surface roughness, thermal gradients or electrical fields on deposition rates and require additional input information. The orientation of the deposition surface influences particle deposition, but few published models have considered deposition rates to both horizontal and vertical surfaces. Particle deposition models have been extensively reviewed 
previously (Sippola, 2002b). In this paper, a state-of-the-art Eulerian model that is based on physically realistic assumptions (Guha, 1997) is compared to measurements made at the duct floor, wall and ceiling of both steel and insulated ducts. This model accounts for particle motion by gravitational settling, Brownian diffusion, turbulent diffusion and turbophoresis. Changes in deposition rates owing to changes in the deposition surface roughness are predicted by a simple method.

\section{Results}

A summary of measured particle and airflow data from each experiment is given in Table 1. Run 7 was performed with the same particle size and air speed as run 6 to evaluate the repeatability of the experiments; all other experiments reflect unique combinations of particle size, air speed, and duct type. Table 1 also reports averages for measured deposition velocities to the duct ceiling $\left(V_{d, c}^{+}\right)$, wall $\left(V_{d, w}^{+}\right)$and floor $\left(V_{d, f}^{+}\right)$of test ducts 1 and 2 in each experiment.

Figure 3 shows measured deposition velocities to the duct ceiling, wall and floor versus air speed for the both the steel and the insulated ducts. Deposition velocities presented in these figures are the average of those measured in test ducts 1 and 2. Measurements made in the steel system at air speeds of 2.2, 5.3 and $9.0 \mathrm{~m} / \mathrm{s}$ are presented as plots of dimensionless deposition velocity $\left(V_{d}^{+}\right)$versus dimensionless relaxation time $\left(\tau^{+}\right)$in Figure $4 \mathrm{a}$ and data collected in the insulated system at air speeds of 2.2, 5.3 and $8.8 \mathrm{~m} / \mathrm{s}$ are likewise presented in Figure $4 \mathrm{~b}$. In these figures, measurements from test ducts 1 and 2 are shown as independent data points. Vertical error bars indicate the variability among deposition velocities quantified on each of the four duct panels for the given surface. These error bars are only shown in cases where they are significantly larger than the data point. 
Measured dimensionless deposition velocities on duct walls at all three air speeds are compared to the vertical-wall deposition data collected by Liu \& Agarwal (1974) in Figure 5a. Wells \& Chamberlain (1967) collected data for particles depositing to a vertical surface covered with fibrous filter paper with a fiber length of $100 \mu \mathrm{m}$. These data are compared to the dimensionless wall deposition velocities measured in the insulated system in Figure $5 \mathrm{~b}$.

Figure 6a compares the model of Guha (1997) to experimental data collected in steel ducts and Figure $6 \mathrm{~b}$ compares the same model applied with a surface roughness value of $180 \mu \mathrm{m}$ to data collected in insulated ducts. The $180-\mu \mathrm{m}$ roughness scale was chosen to best match model predictions with the deposition data.

\section{Discussion}

Figure 3 clearly shows that deposition velocities to duct ceiling, wall and floor surfaces increase with increases in both particle size and air speed. These trends are visible in both systems, but are weaker in the insulated system. For a given air speed, increases in particle diameter from 1 to $16 \mu \mathrm{m}$ increased deposition velocities to steel duct ceilings by factors in the range 40-180. For steel duct walls and floors, the same factors were in the ranges $160-920$ and 200-420, respectively. In steel ducts, the influence of particle size on deposition rates was weakest at the duct ceiling surface and strongest at the duct wall. For a given particle size, increasing the air speed from 2.2 to $9.0 \mathrm{~m} / \mathrm{s}$ increased deposition velocities to the duct ceiling by factors in the range 5-120. The same factors were in the ranges 6-50 and 1.5-4 for duct walls and floors, respectively. The influence of air speed on deposition rates in steel ducts was strongest for deposition to the duct ceiling and weakest for the duct floor.

For a given air speed in insulated ducts, increases in particle diameter from 1 to $16 \mu \mathrm{m}$ increase deposition rates to duct ceilings by factors in the range 10-30. These same factors for 
the duct wall and floor are in the ranges $30-40$ and 30-100, respectively. For a given particle size, increasing the air speed from 2.2 to $8.8 \mathrm{~m} / \mathrm{s}$ increased deposition rates to insulated duct ceilings by factors in the range 20-50. The same factors were in the ranges $15-50$ and 5-20 for insulated duct walls and floors, respectively. The weaker influence of particle size and air speed on deposition rates to insulated ducts as compared to steel ducts likely reflects the importance of insulation roughness elements in determining particle deposition rates. This observation is in accord with most deposition model predictions that account for surface roughness.

Deposition velocities to duct ceilings and walls were greatly enhanced in insulated ducts as compared to steel ducts. Deposition to the duct floor increased only slightly from steel ducts to insulated ducts. For 1-5 $\mu \mathrm{m}$ particles, deposition rates to ceilings of insulated ducts were 80800 times higher than those to ceilings of steel ducts at the same air speed. For duct walls and floors, the same factors were in the ranges 15-200 and 0.8-20, respectively. Quantitative comparisons between the two systems are confounded at the larger particle sizes because the experiments were conducted at different particle sizes in the two systems.

The importance of deposition surface orientation for determining deposition rates to steel ducts is apparent from Figure 4a. For a given particle size and air speed, the deposition rate to the steel duct floor was always greater than the deposition rate to the duct wall, which, in turn, was always greater than the rate to the ceiling. Data at the lowest air speed show deposition rates to the duct floor that were 2-3 orders of magnitude greater than rates to the ceiling and 1-2 orders of magnitude greater than rates to the wall. In steel ducts, the ratios of deposition rates to the duct floor, relative to the wall or ceiling, decreased with increasing air speed.

Data at the lowest air speed in the insulated ducts, presented in Figure 4b, show deposition rates to the duct ceiling, wall and floor to be nearly equal for the smallest particle size. 
For larger particles, differences in deposition rates to the different surfaces are apparent. Owing to the influence of gravity, deposition rates to the floor are greater than to the wall or ceiling for 3-13 $\mu \mathrm{m}$ particles. At the lowest air speed, differences in deposition velocities to the duct ceiling, wall and floor are much smaller in the insulated system than in the steel system. Data in insulated ducts at the two higher air speeds in Figure $4 \mathrm{~b}$ show nearly equal deposition rates to the ceiling, wall and floor surfaces for all particle sizes. Measured dimensionless deposition velocities to the floor surface are usually slightly higher than to the wall or ceiling, but differences are far smaller than observed in steel ducts. Particles deposited in a nearly uniform manner in the insulated ducts at the two higher air speeds; this suggests that roughness elements associated with the insulation play a dominant role in controlling deposition at these air speeds. Measured values of $V_{d}^{+}$at the two higher air speeds show good agreement with values from Liu \& Agarwal (1974) in Figure 5a, but our data for $U_{\text {ave }}=2.2 \mathrm{~m} / \mathrm{s}$ lie above all other data. When plotted as $V_{d}^{+}$versus $\tau^{+}$, data for deposition to vertical walls collected at different air speeds are expected to fall on a single curve. The data of Liu \& Agarwal were collected at a friction velocity of about $74 \mathrm{~cm} / \mathrm{s}$. The lack of agreement of the low-air-speed data with the other data is possibly attributable to the low friction velocity in those experiments relative to the others. A similar increase in values of $V_{d}^{+}$with a decrease in friction velocity was observed in the simulations of Zhang \& Ahmadi (2000) at low friction velocities.

In Figure 5b, comparing measured deposition rates to insulated duct walls to similar data from the literature, Wells \& Chamberlain's (1967) data lie above the data measured in the insulated system, but similar trends are observed in both data sets. Both exhibit a similar slope for data with $\tau^{+}<1$ and a leveling of the trend for values of $\tau^{+}>1$. Wells and Chamberlain observed a marked increase in deposition rates to a fibrous surface compared to a smooth 
metallic surface; a similar deposition enhancement was observed in the current experiments in insulated ducts as compared to steel ducts (compare Figure 4a with Figure 4b).

In Figure 6a, model-measurement agreement is good for data at the duct floor, but the model substantially underpredicts measurements at the duct wall and drastically underpredicts measurements at the duct ceiling. In Figure $6 \mathrm{~b}$, the model is applied with a roughness of $180 \mu \mathrm{m}$ and the model predicts nearly uniform deposition to duct floors, walls and ceilings. At this high roughness level, the model exhibits reasonable estimates of the magnitude and trends observed in the data; however, it significantly overpredicts deposition rates of the smallest $(1 \mu \mathrm{m})$ particles.

\section{Conclusions}

Particle deposition rates to the ceiling, walls and floor of steel and insulated horizontal ducts have been measured for a range of particle sizes and air speeds. These deposition rates, measured in ducts with fully developed turbulent flow, are summarized in Table 1 . The experiments used real HVAC materials and deposition to duct surfaces with different orientations was directly measured.

In steel ducts, deposition rates were much greater to the floor than to the walls or ceiling for all particle sizes and air speeds. Increasing air speed within the range $2.2-9.0 \mathrm{~m} / \mathrm{s}$ led to increased deposition rates to steel surfaces of all orientations, as did increasing particle size within the range 1-16 $\mu \mathrm{m}$. For a given particle size, air speed and duct surface, deposition rates were higher, often much higher, in insulated ducts than in steel ducts; this was especially true for wall and ceiling surfaces. Deposition to duct floors at the lowest air speed of $2.2 \mathrm{~m} / \mathrm{s}$ was the only case in which deposition rates in insulated ducts were not significantly enhanced compared to rates in steel ducts. Deposition in the insulated ducts was nearly uniform to floor, wall and ceiling surfaces, especially at air speeds of 5.3 and $8.8 \mathrm{~m} / \mathrm{s}$. Increasing air speed and particle size 
led to increases in deposition rates to insulated duct surfaces, but the effect was less dramatic than observed in steel systems. The rough and fibrous character of the exposed insulation inside an insulated duct appears to be a strong factor influencing particle deposition.

These experiments have addressed particle deposition in ventilation ducts with turbulent flow. The reported data illustrate clear trends and establish expected magnitudes of particle deposition rates under a variety of duct conditions. These data should be useful for improving our ability to predict particle losses in portions of ventilation duct runs with fully developed turbulent flow. Flow paths through ventilation ducts are commonly complex; there are many circumstances where the turbulent flow profile is disturbed or not fully developed and these conditions are likely to influence particle deposition to duct surfaces. Investigations of particle deposition downstream of flow disturbances and within duct bends is needed for a more complete understanding of deposition in ventilation ducts.

\section{Acknowledgements}

This work was supported by the Office of Research and Development, Office of Nonproliferation and National Security, US Department of Energy under Contract No. DEAC03-76SF00098.

\section{References}

Browne, L. W. B. (1974). Deposition of Particles on Rough Surfaces during Turbulent Gas-Flow in a Pipe, Atmos. Environ. 8: 801-816.

Chamberlain, A. C., Garland, J. A., and Wells, A. C. (1984). Transport of Gases and Particles to Surfaces with Widely Spaced Roughness Elements, Boundary Layer Meteor. 29: 343-360. 
El-Shobokshy, M. S. (1983). Experimental Measurements of Aerosol Deposition to Smooth and Rough Surfaces, Atmos. Environ. 17: 639-644.

Fan, F.-G., and Ahmadi, G. (1993). A Sublayer Model for Turbulent Deposition of Particles in Vertical Ducts with Smooth and Rough Surfaces, J. Aerosol Sci. 24: 45-64.

Guha, A. (1997). A Unified Eulerian Theory of Turbulent Deposition to Smooth and Rough Surfaces, J. Aerosol Sci. 28: 1517-1537.

Kvasnak, W., Ahmadi, G., Bayer, R., and Gaynes, M. (1993). Experimental Investigation of Dust Particle Deposition in a Turbulent Channel Flow. J. Aerosol Sci. 24: 795-815.

Lai, C. K. (1997). An Experimental Study of the Deposition of Aerosol on Rough Surfaces and the Implications for Indoor Air Quality Control, Ph. D. Dissertation, Imperial College, London, England.

Liu, B. Y. H., and Agarwal, J. K. (1974). Experimental Observation of Aerosol Deposition in Turbulent Flow. Aerosol Sci. 5: 145-155.

Sehmel, G. A. (1970). Turbulent Deposition of Monodisperse Particles on Simulated Grass. In Assessment of Airborne Particles: Fundamentals, Applications, and Implications to Inhalation Toxicity, edited by Mercer, T. T., Morrow, P. E., and Stöber, W.. Charles C. Thomas, Springfield, Illinois.

Sehmel, G. A. (1973). Particle Eddy Diffusivities and Deposition Velocities for Isothermal Flow and Smooth Surfaces. Aerosol Sci 4: 125-138.

Sippola, M. R. (2002a). Particle Deposition in Ventilation Ducts, Ph. D. Dissertation, University of California, Berkeley, California, USA. 
Sippola, M. R. (2002b). Particle Deposition from Turbulent Flow: Review of Published Research and Its Applicability to Ventilation Ducts in Commercial Buildings, $L B N L-51432$, Berkeley, California: Lawrence Berkeley National Laboratory.

Sippola, M. R, and Nazaroff, W. W. (2003). Modeling Particle Loss in Ventilation Ducts, to be published in Atmos. Environ.

Wells, A. C., and Chamberlain, A. C. (1967). Transport of Small Particles to Vertical Surfaces. Brit. J. Appl. Phys. 18: 1793-1799.

Zhang, H., and Ahmadi, G. (2000). Aerosol Particle Transport and Deposition in Vertical and Horizontal Turbulent Duct Flows. J Fluid Mech. 406: 55-80. 
Table 1

Measured air velocities, particle sizes and dimensionless deposition velocities to the ceiling $\left(V_{d, c}^{+}\right)$, wall $\left(V_{d, w}^{+}\right)$and floors $\left(V_{d, f}^{+}\right)$for all experiments in test ducts 1 and $2 .{ }^{a}$

\begin{tabular}{|c|c|c|c|c|c|c|c|c|}
\hline $\begin{array}{c}\text { Run \# } \\
(-)\end{array}$ & $\begin{array}{c}\text { Duct } \\
\text { system }\end{array}$ & $\begin{array}{c}U_{\text {ave }} \\
\left(\mathrm{m} \mathrm{s}^{-1}\right)\end{array}$ & $\begin{array}{c}u^{*} \\
\left(m^{-1} s^{-1}\right)\end{array}$ & $\begin{array}{c}d_{p} \\
(\mu \mathrm{m})\end{array}$ & $\begin{array}{c}\tau^{+} \\
(-)\end{array}$ & $\begin{array}{c}V_{d, c}^{+} \\
(-)\end{array}$ & $\begin{array}{c}V_{d, w}^{+} \\
(-)\end{array}$ & $\begin{array}{c}V_{d, f}^{+} \\
(-)\end{array}$ \\
\hline 1 & steel & 2.2 & 0.12 & 1.0 & $4.6 \times 10^{-3}$ & $2.4 \times 10^{-6}$ & $1.1 \times 10^{-5}$ & $3.9 \times 10^{-4}$ \\
\hline 2 & steel & 2.2 & 0.12 & 2.8 & 0.028 & $2.2 \times 10^{-5}$ & $6.8 \times 10^{-5}$ & $2.7 \times 10^{-3}$ \\
\hline 3 & steel & 2.1 & 0.12 & 5.2 & 0.098 & $4.2 \times 10^{-5}$ & $3.1 \times 10^{-4}$ & 0.011 \\
\hline 4 & steel & 2.2 & 0.13 & 9.1 & 0.27 & $8.1 \times 10^{-5}$ & $7.6 \times 10^{-4}$ & 0.028 \\
\hline 5 & steel & 2.2 & 0.12 & 16 & 0.72 & $9.0 \times 10^{-5}$ & $1.7 \times 10^{-3}$ & 0.072 \\
\hline 6 & steel & 5.3 & 0.28 & 1.0 & 0.023 & $4.5 \times 10^{-6}$ & $2.2 \times 10^{-5}$ & $2.1 \times 10^{-4}$ \\
\hline 7 & steel & 5.2 & 0.26 & 1.0 & 0.022 & $5.5 \times 10^{-6}$ & $2.5 \times 10^{-5}$ & $2.0 \times 10^{-4}$ \\
\hline 8 & steel & 5.2 & 0.26 & 3.1 & 0.16 & $2.0 \times 10^{-5}$ & $5.0 \times 10^{-5}$ & $1.5 \times 10^{-3}$ \\
\hline 9 & steel & 5.4 & 0.27 & 5.2 & 0.48 & $4.8 \times 10^{-5}$ & $3.5 \times 10^{-4}$ & $8.2 \times 10^{-3}$ \\
\hline 10 & steel & 5.3 & 0.28 & 9.8 & 1.5 & $6.4 \times 10^{-5}$ & $1.0 \times 10^{-3}$ & 0.019 \\
\hline 11 & steel & 5.3 & 0.28 & 16 & 3.6 & $4.5 \times 10^{-4}$ & 0.015 & 0.072 \\
\hline 12 & steel & 9.0 & 0.45 & 1.0 & 0.062 & $1.7 \times 10^{-5}$ & $2.6 \times 10^{-5}$ & $1.9 \times 10^{-4}$ \\
\hline 13 & steel & 9.0 & 0.42 & 3.1 & 0.42 & $2.9 \times 10^{-5}$ & $1.1 \times 10^{-4}$ & $1.2 \times 10^{-3}$ \\
\hline 14 & steel & 8.8 & 0.44 & 5.4 & 1.4 & $1.5 \times 10^{-4}$ & $1.4 \times 10^{-3}$ & 0.013 \\
\hline 15 & steel & 9.2 & 0.46 & 8.7 & 3.2 & $9.4 \times 10^{-4}$ & $3.5 \times 10^{-3}$ & 0.026 \\
\hline 16 & steel & 9.1 & 0.45 & 15 & 8.8 & $2.9 \times 10^{-3}$ & 0.024 & 0.080 \\
\hline 17 & insulated & 2.2 & 0.16 & 1.0 & $7.8 \times 10^{-3}$ & $4.1 \times 10^{-4}$ & $3.7 \times 10^{-4}$ & $5.3 \times 10^{-4}$ \\
\hline 18 & insulated & 2.2 & 0.16 & 3.0 & 0.054 & $1.3 \times 10^{-3}$ & $1.1 \times 10^{-3}$ & $2.6 \times 10^{-3}$ \\
\hline 19 & insulated & 2.2 & 0.16 & 5.3 & 0.17 & $2.7 \times 10^{-3}$ & $3.4 \times 10^{-3}$ & $7.2 \times 10^{-3}$ \\
\hline 20 & insulated & 2.2 & 0.16 & 8.4 & 0.39 & $4.0 \times 10^{-3}$ & $5.4 \times 10^{-3}$ & 0.016 \\
\hline 21 & insulated & 2.2 & 0.16 & 13 & 0.81 & $5.4 \times 10^{-3}$ & 0.014 & 0.052 \\
\hline 22 & insulated & 5.3 & 0.37 & 1.0 & 0.043 & $1.1 \times 10^{-3}$ & $1.2 \times 10^{-3}$ & $1.2 \times 10^{-3}$ \\
\hline 23 & insulated & 5.2 & 0.37 & 2.9 & 0.28 & $7.2 \times 10^{-3}$ & $6.1 \times 10^{-3}$ & $7.6 \times 10^{-3}$ \\
\hline 24 & insulated & 5.2 & 0.37 & 4.9 & 0.80 & 0.022 & 0.019 & 0.023 \\
\hline 25 & insulated & 5.3 & 0.38 & 8.2 & 2.1 & 0.034 & 0.038 & 0.046 \\
\hline 26 & insulated & 5.3 & 0.38 & 13 & 4.5 & 0.036 & 0.041 & 0.057 \\
\hline 27 & insulated & 8.9 & 0.62 & 1.0 & 0.12 & $2.3 \times 10^{-3}$ & $2.2 \times 10^{-3}$ & $2.4 \times 10^{-3}$ \\
\hline 28 & insulated & 8.7 & 0.62 & 2.8 & 0.72 & 0.015 & 0.015 & 0.016 \\
\hline 29 & insulated & 8.9 & 0.62 & 5.0 & 2.3 & 0.030 & 0.030 & 0.034 \\
\hline 30 & insulated & 8.9 & 0.64 & 8.4 & 6.0 & 0.047 & 0.051 & 0.060 \\
\hline 31 & insulated & 8.9 & 0.64 & 13 & 12 & 0.051 & 0.055 & 0.070 \\
\hline
\end{tabular}

${ }^{\mathrm{a}}$ Symbol definitions: $U_{\text {ave }}$ - mean air speed through duct; $u^{*}$ - friction velocity; $d_{p}$ - particle diameter; $\tau^{+}$- dimensionless relaxation time (see equation (6)); $V_{d, c}^{+}-$dimensionless deposition velocity to duct ceiling; $V_{d, w}^{+}-$dimensionless deposition velocity to duct wall; $V_{d, f}^{+}$ — dimensionless deposition velocity to duct floor (see equation (2)). 


\section{Figure Captions}

Figure 1. Schematic diagram of the experimental apparatus (not to scale).

Figure 2. Schematic diagram showing the locations of panels cut out of a straight test duct for determination of the particle flux. Panels on the ceiling and wall are shown. Panels were similarly spaced and labeled on the duct floor.

Figure 3. Average measured deposition velocity to the ceiling, wall and floor versus air speed in the a) steel and b) insulated systems. Particle diameters are nominal; see Table 1 for more accurate specification.

Figure 4. Measured dimensionless deposition velocities $\left(V_{d}^{+}\right)$versus dimensionless relaxation time $\left(\tau^{+}\right)$for the floor, wall and ceiling in the a) steel and b) insulated systems at three air speeds.

Figure 5. Comparison of dimensionless deposition velocity to the wall $\left(V_{d, w}{ }^{+}\right)$versus dimensionless relaxation time $\left(\tau^{+}\right)$measured at three air speeds in the a) steel and b) insulated system and as reported in the literature (Liu \& Agarwal, 1974; Wells \& Chamberlain, 1967).

Figure 6. Comparison of the predictions from the deposition model of Guha (1997) to data collected in a) steel and b) insulated ducts with $U_{\text {ave }}=5.3 \mathrm{~m} / \mathrm{s}$. The parameter $k$ represents the surface roughness length scale; the choice of $180 \mu \mathrm{m}$ was made to optimize the agreement between model predictions and measurements. 


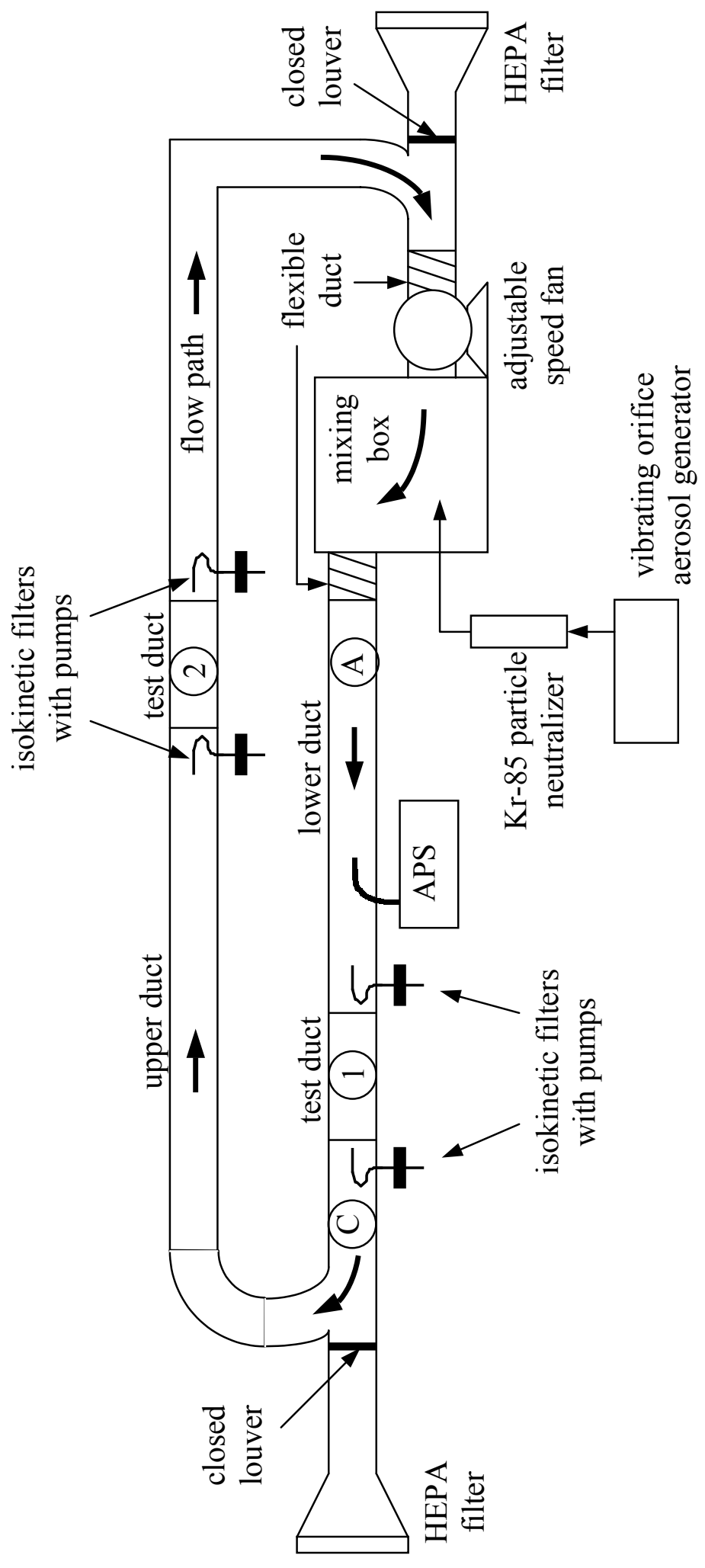

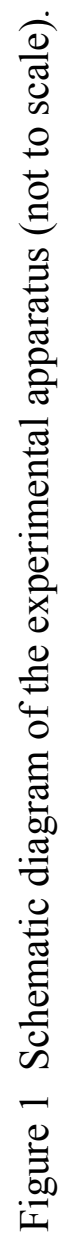




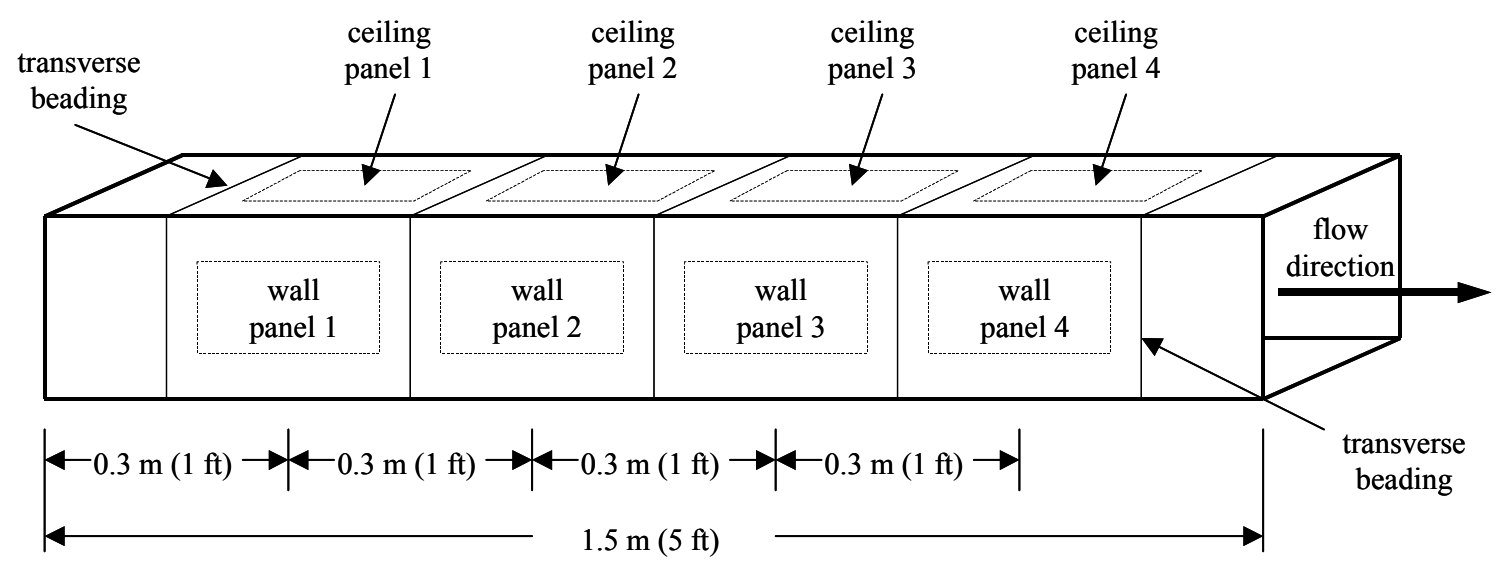

Figure 2 Schematic diagram showing the locations of panels cut out of a straight test duct for determination of the particle flux. Panels on the ceiling and wall are shown. Panels were similarly spaced and labeled on the duct floor. 

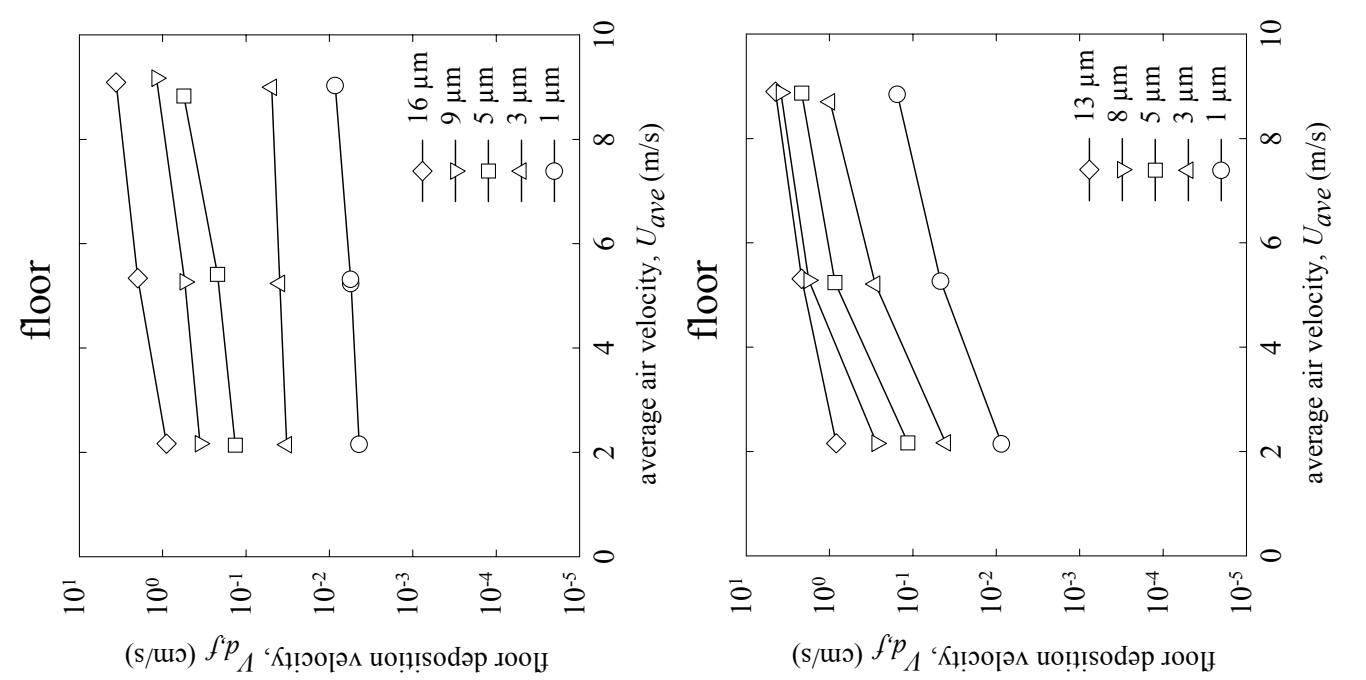

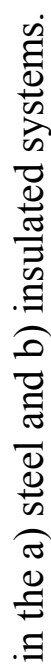
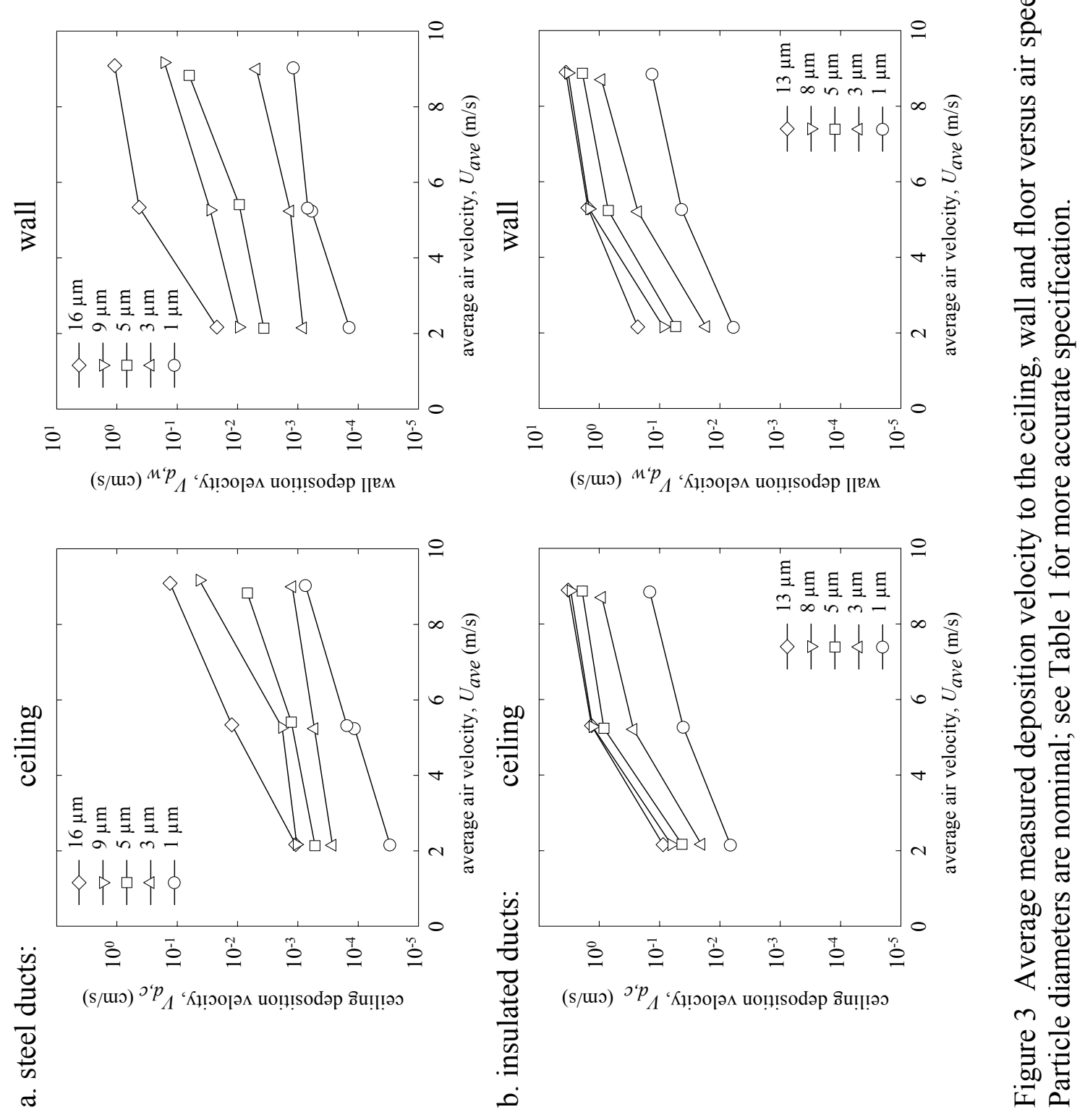


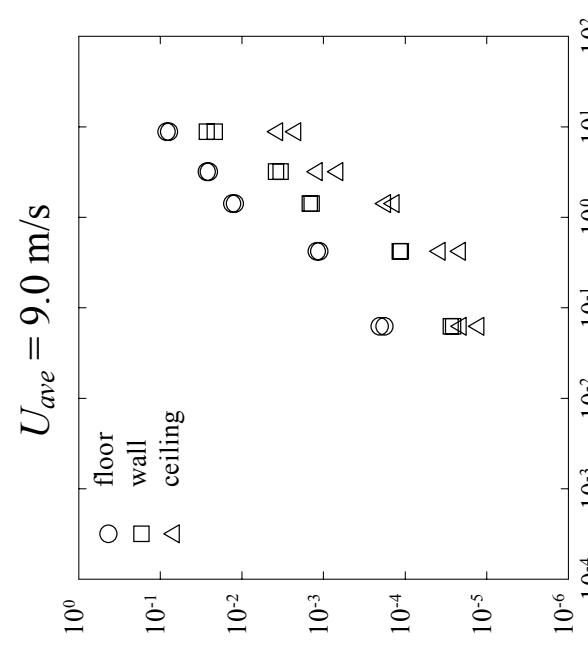

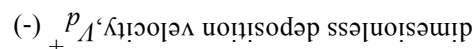

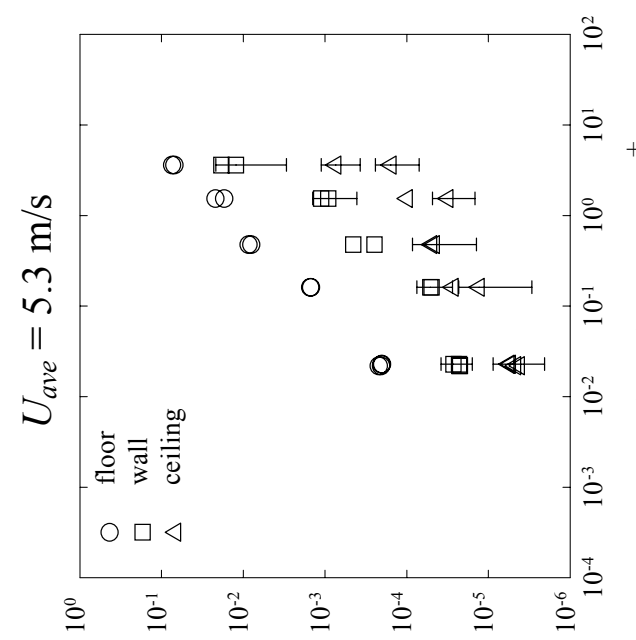

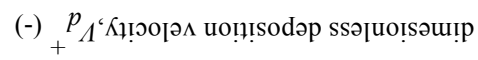

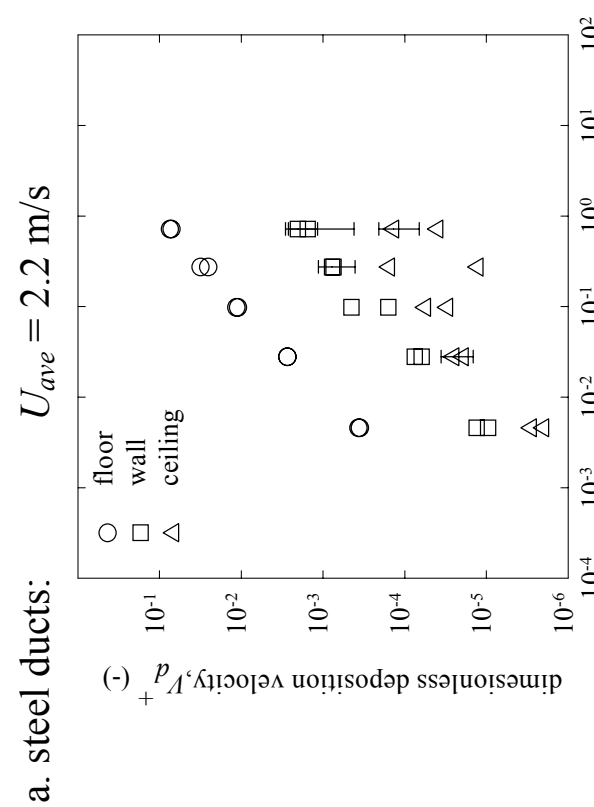

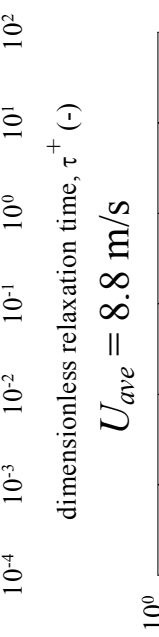

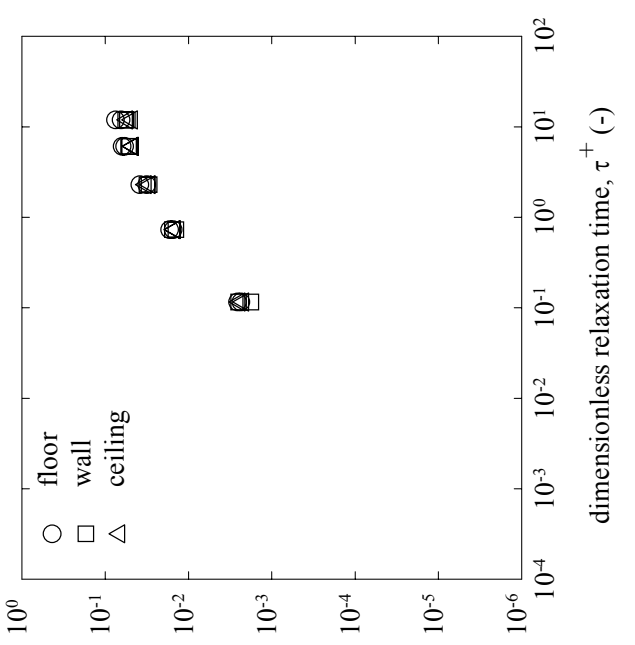

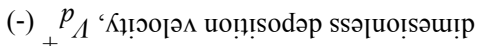

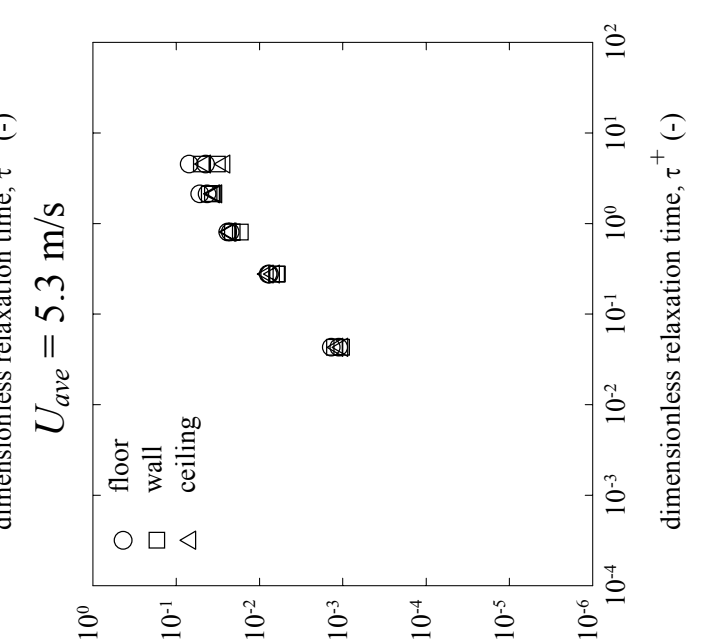

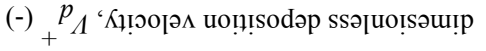

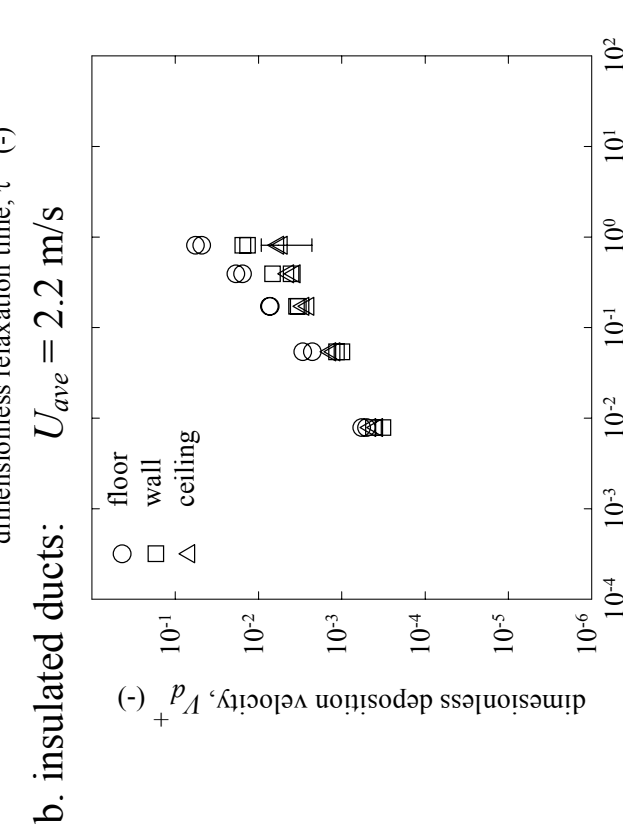

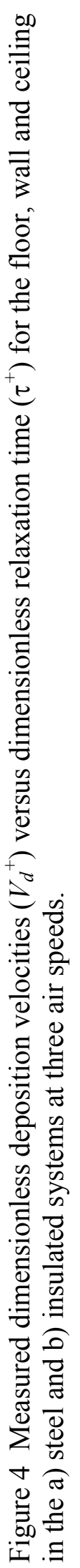


a. steel ducts

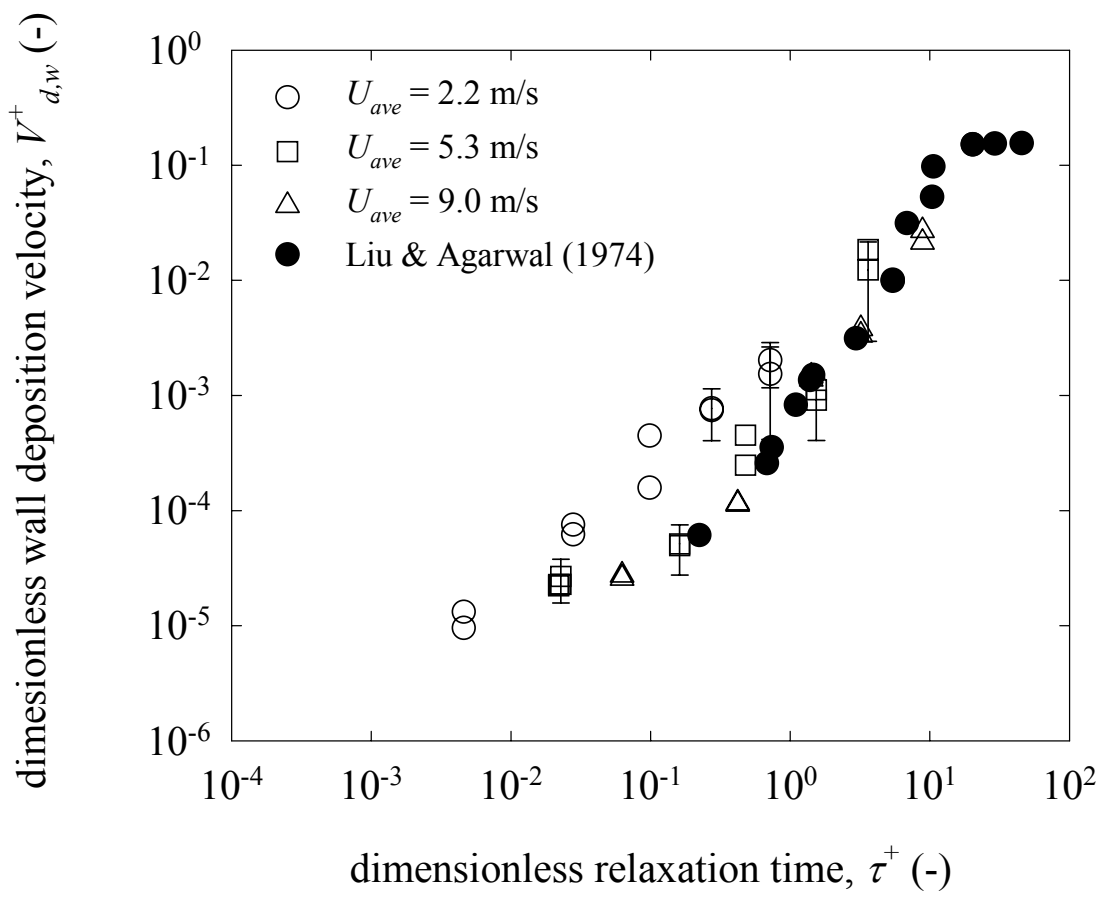

b. insulated ducts

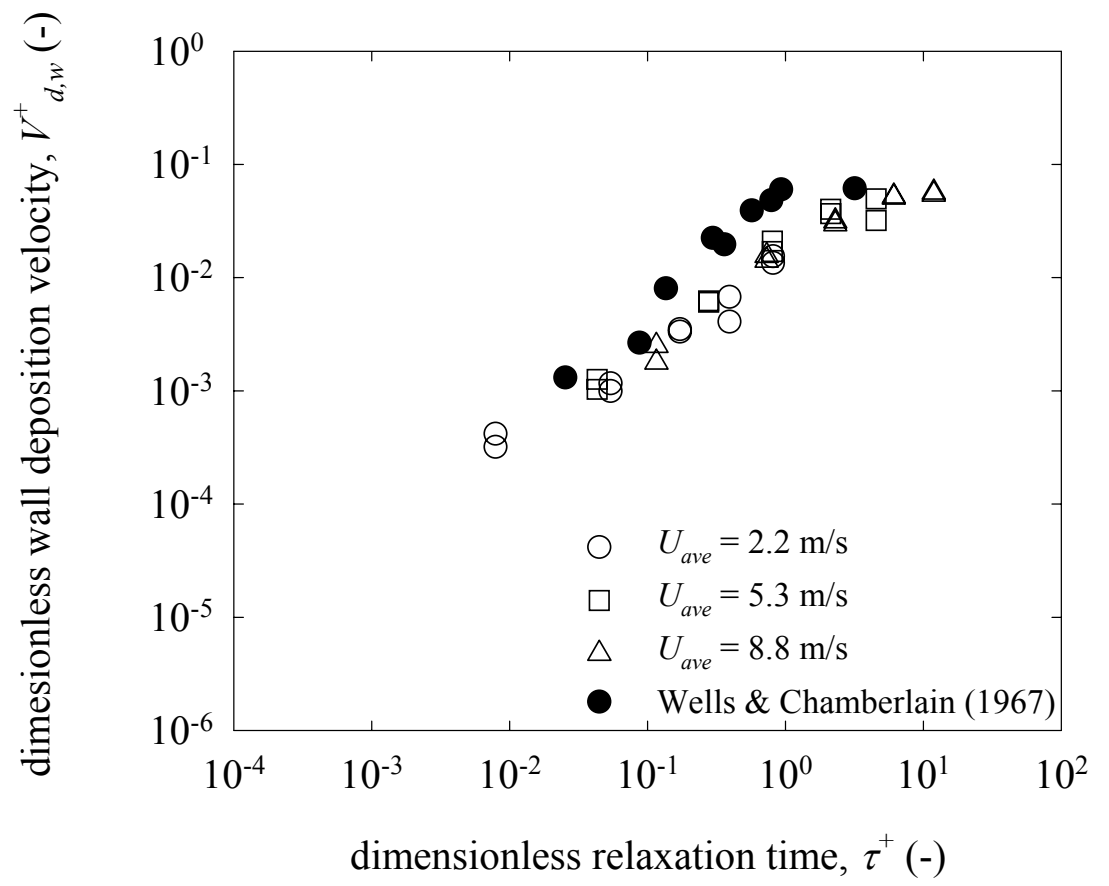

Figure 5 Comparison of dimensionless deposition velocity to the wall $\left(V_{d, w}{ }^{+}\right)$versus dimensionless relaxation time $\left(\tau^{+}\right)$measured at three air speeds in the a) steel and b) insulated system and as reported in the literature (Liu \& Agarwal, 1974; Wells \& Chamberlain, 1967). 
a. steel ducts

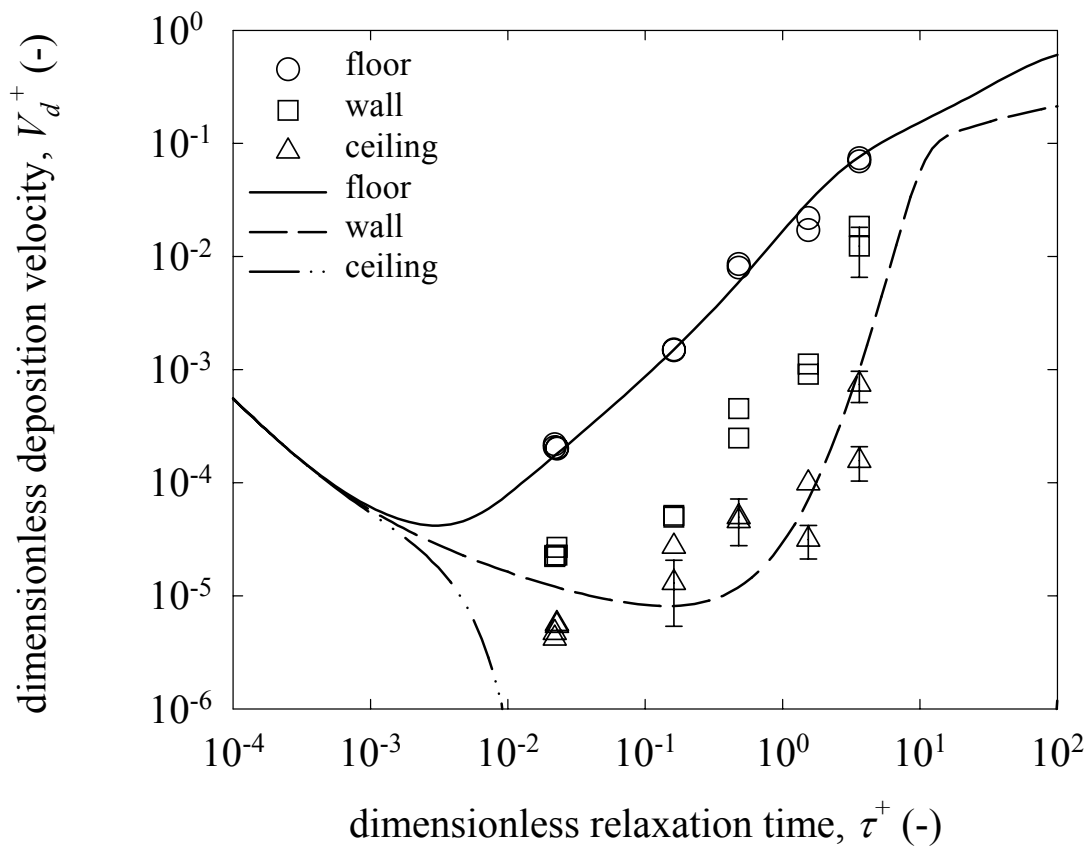

b. insulated ducts

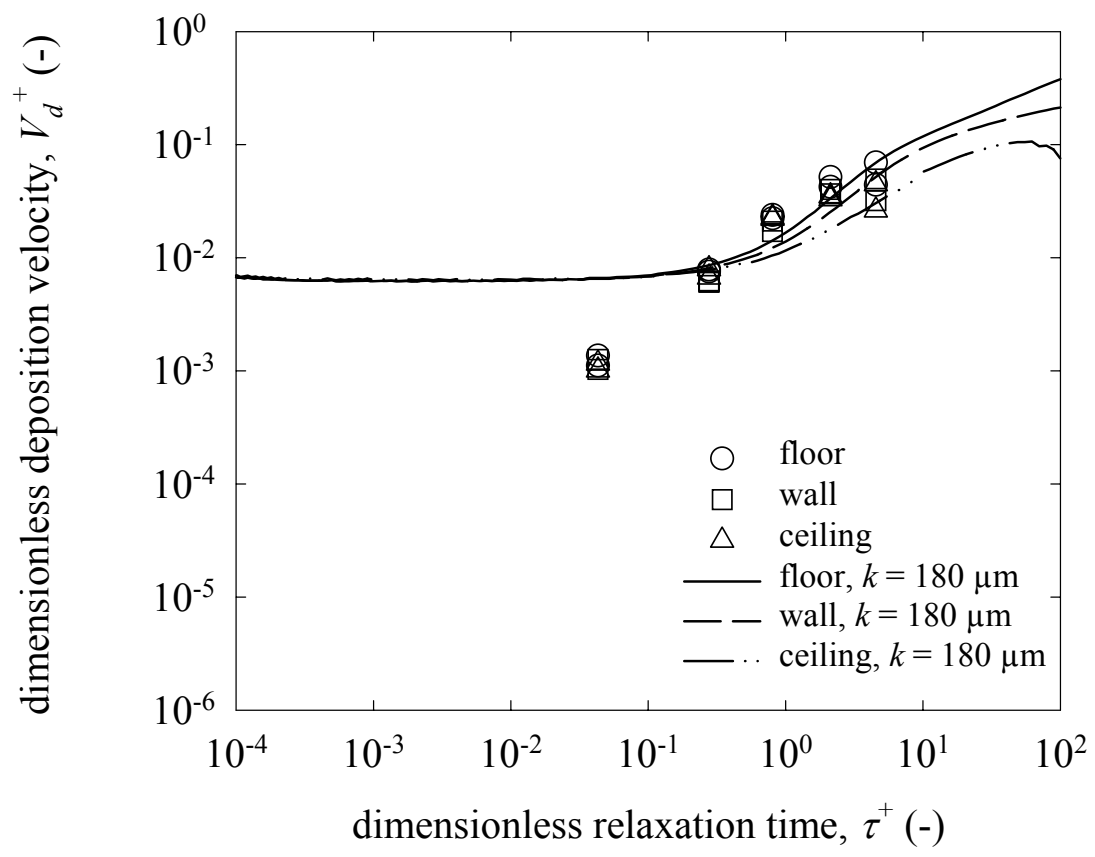

Figure 6 Comparison of the predictions from the deposition model of Guha (1997) to data collected in a) steel and b) insulated ducts with $U_{\text {ave }}=5.3 \mathrm{~m} / \mathrm{s}$. The parameter $k$ represents the surface roughness length scale; the choice of $180 \mu \mathrm{m}$ was made to optimize the agreement between model predictions and measurements. 